\title{
Contact force control of a Three-dimensional Flexible Manipulator
}

\author{
Minoru Sasaki 1,2*, Shunta Ito 2, Daiki Maeno 2, Waweru Njeri 1,2,3, Joseph Muguro 2,3, Titus \\ Mulembo ${ }^{1,2,4}$, Kojiro Matsushita ${ }^{1,2}$ \\ 1 Intelligent Production Technology Research \& Development Center for Aerospace (IPTeCA), Gifu University, 1- \\ 1 Yanagido, Gifu, 501-1193, Japan. \\ 2 Department of Mechanical Engineering, Faculty of Engineering, Gifu University, 1-1 Yanagido, Gifu, 501-1193, \\ Japan. \\ 3 Department of Electrical \& Electronic Engineering, Dedan Kimathi University of Technology, Nyeri, Kenya \\ 4 Department of Mechatronic Engineering, Dedan Kimathi University of Technology, Nyeri, Kenya \\ * Correspondence: sasaki@gifu-u.ac.jp, Tel.: +81582932541
}

\begin{abstract}
Flexible manipulators using lightweight materials offer the advantage of low energy consumption. However, to enable the manipulators to work in contact environments, force control of such arms with elastically deforming links in 3-dimension motion is a topic of interest. A simple proportional contact force controller for a constrained flexible manipulator capable of threedimensional motion is presented here. This controller uses a conversion formula obtained empirically and the experimental results showed the effectiveness of the proposed contact force controller. To realize this, the manipulator was operated with the tip of the second link restrained, then, the time response of the root strain, the joint angles, and the robot arm's tip contact force was used to derive the relational expression between these three quantities. The relational expression was verified by conducting a target contact force tracking experiment by inputting the angle from the relational expression. Contact force control using the strain feedback and force feedback methods was proposed and the control algorithms were verified through experiments. Set-point force values of $-1 \mathrm{~N},-2 \mathrm{~N}$, and $-3 \mathrm{~N}$ were effectively tracked using both the controllers.
\end{abstract}

Keywords: Contact force control; Three-dimensional; Flexible Manipulator; Strain feedback; Force Feedback

\section{Introduction}

In a conventional robot arm, increasing the structural rigidity improves the accuracy of the robot arm's tip position. However, an increase in rigidity results to increase in weight and energy consumption of the robot. Further, the material costs incurred in the design increases. Therefore, energy-saving, lowering design costs and enabling high-speed operation are some of the technical issues affecting the application of the industrial robot arm. To address this, low weight, low-cost flexible manipulators have become a subject of focus. However, since the rigidity of the flexible arm is compromised, vibration due to elastic deformation of the arm itself cannot be ignored. Further, conventional control methods for the rigid body model do not consider position compensation or vibration suppression for both the position error due to the elastic deformation and the effects of elastic vibrations, respectively. Therefore, the established research results for the rigid robot arms cannot be applied as they are [1]-[3]. Research on vibration control of elastically deforming flexible manipulators is actively being conducted [4], [5]. In literature, a one-link arm is called a flexible arm whereas a two-or-more link arm is called a flexible manipulator.

When a robot arm performs various tasks, it is necessary to measure the force applied to an object by the robot arm's tip to ensure that the appropriate contact force is applied. For a flexible arm to be applied in a factory setting, many tasks exist, such as screwing, part assembly, painting, transporting objects. For all these tasks, it is important to perform trajectory control, elastic vibration control, and 
contact force control for the flexible manipulator. A control strategy where the displacement of the link is directly measured and is fed back has gained attention [6]-[8].

Kojima et al. [9] used a constrained horizontal two-link manipulator where the first link was rigid, and the second link was flexible. The authors sought to keep a constant contact force at the tip of their robot arm, and they implemented force control by strain signal feedback. First, the equation of motion of the dynamic system was derived, and the control command value of the joint angle when elastic deformation was not considered was derived using inverse kinematics. After that, simulations and experiments of the two contact force control approaches in a non-motion and motion state were performed. However, it was concluded that the strain feedback control method was a more effective means because the disturbance due to non-linear force could not be fully taken into consideration.

Endo et al. [10]-[12] used a simple boundary feedback controller consisting of a bending moment at the base of a flexible arm, and its time derivative, and implemented an analytical model for the force control problem for a constrained flexible arm. The exponential stability of the closed-loop system using this simple feedback control was analytically demonstrated therein. The authors performed simulations to examine the effectiveness of the proposed controller, but the verification of the performance of the proposed controller and the method of adjusting the feedback gain remained a problem. Honda et al. independently worked on force control of a constrained flexible arm. A simple boundary feedback controller (moment-PD) was employed and the performance of the controller was verified through experiments. Then, Sasaki et al. further proposed a control method using the neural network as a gain adjustment method on Honda's model, and they proved the effectiveness of the control performance using an actual machine [13], [14]. Ferretti et al. [15] used 6 degrees of freedom (DOF) rigid arm and an ATI type force sensor at the tip of the robot arm. They only let the Joint 5 move which pushed against a stiff surface. They used a lumped 2 mass model to represent the moving robot arm. They successfully used integral force control with velocity feedback to prove the robustness of their controller, both by simulation and experiment.

In this paper, we used a spatial two-link flexible manipulator to study force control. Many studies have been on modeling and control of a one-link arm and a few have been about the two-link flexible manipulator. Also, as pointed out earlier, a rigid arm has its demerits as compared to a flexible arm. In as much as the 3D two-link flexible arm is more practical, and the derivation of the infinite model of this manipulator for vibration control research would be feasible, little research has been done due to its complexity. Further, such analytical models would require very fast computers having large memory storage considering the number of calculations involved. Here, feedback of both link strain amount and tip force will be presented and analyzed. The reason for opting for direct force feedback is that analytical models would not be required hence the computation cost would be lowered. Our originality lies in the fact that this is the first report which implements and compares both force and strain feedback for a two-link flexible manipulator. Also, unlike the reported two-link flexible robot arm studies by Kojima et al [9], for our case, both links are flexible.

To this end, the relation between the contact force and the strain amount was obtained by Hooke's law, within the proportional limit. We hypothesize that this relationship holds for the range of the joint angles that we carry the experiments on. To achieve our goal, the tip of the robot arm is constrained to operate, and the relational expressions of the contact force at the tip, the strain, and the operating joint angle are empirically derived. The target contact force tracking experiment was performed by inputting an angle from the relational expression. After that, the contact force is estimated from the amount of strain. The performance of the force controllers is verified experimentally. Results from this work would further give insights into the possibilities of implementing such spatial flexible manipulators in high-speed industrial applications.

The rest of the paper is organized as follows: Section 2 presents the flexible arm and the experimental setup for the control experiment. The formulation and experimental validation of the relational expression of the robot arm parameters are presented. The expressions were tested against uniformly incremental force and step forces. Section 3 shows the two proposed control schemes, based on strain and force feedback. Here, the setpoint for both the controllers is force. Section 4 presents the experimental results for the performance of the two force control strategies. Table 1 gives the nomenclatures used in this paper. 
Table 1. Nomenclature.

\begin{tabular}{ll}
\hline Notation & Full name \\
\hline DOF & Degrees of freedom \\
FB & Feedback \\
PD & Proportional plus Derivative \\
DC & Direct current \\
DAC & Digital to analog converter \\
ADC & Analog to digital converter \\
\hline
\end{tabular}

\section{Experimental}

This section describes the flexible manipulator, the force sensor, the electrical equipment used for the experiment, and the experimental conditions.

\subsection{The flexible manipulator}

The 3D spatial flexible manipulator that was used is as shown in Figure 1a having two links and three joints, hence three degrees of freedom. Joint 1 and Joint 2 are located at the base. Joint 3 is between link 1 and link 2 . Joint 2 and Joint 3 rotate in the elevation direction while joint 1 rotates in the azimuth direction giving the manipulator a 3D sweep. The first link is made of stainless-steel rod and the second link is an aluminum rod. Each joint is driven by a DC servomotor and strain gauges are attached at the root of each link to measure strain in torsional and bending senses, and not longitudinally along the links neutral axis. A force sensor (BL NANO sensor manufactured by BL Autotech) was attached to the tip of the robot arm and is shown in Figure 1b. The transducer of this sensor contains a Y-shaped strain element, and 6 sets of strain gauges are attached to the bridge. Measurement is performed by converting strain into electrical resistance with this gauge and amplifying it with an amplification unit. Moreover, since the amplifier unit uses the differential output method, it has the advantages of high speed, stability, and resistance to noise compared to the single-ended output method.

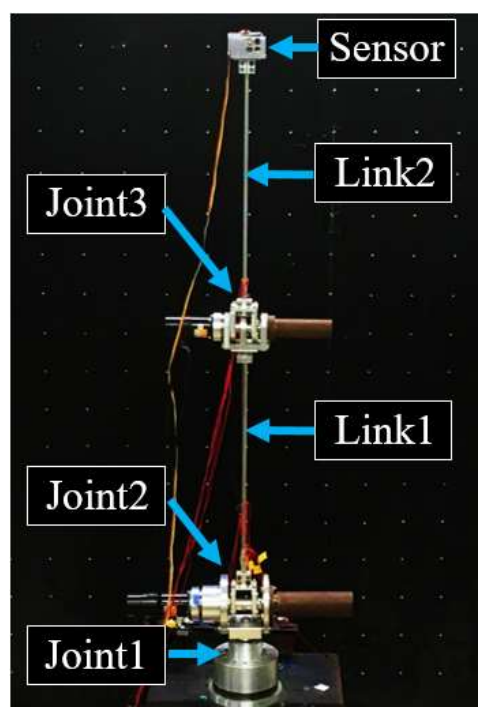

(a)

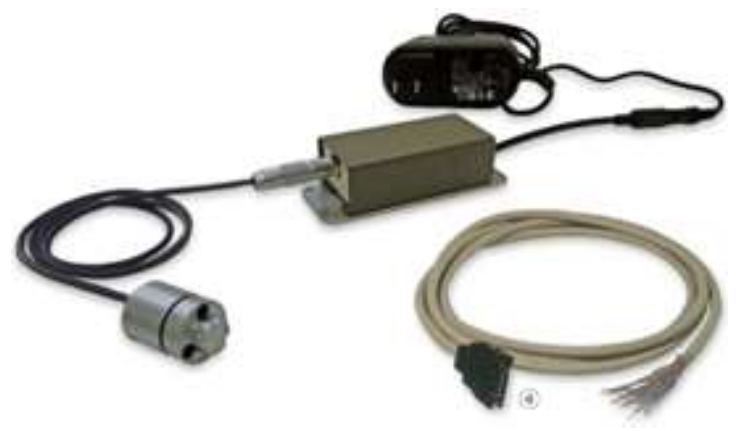

(b)

Figure 1. The experimental arm system (a) The 3D flexible manipulator; (b) Force sensor unit.

\subsection{Control experiment setup}

The DC motor is driven by a DC motor servo driver which gets their signal from the dSPACE board. Feedback angles signals from the optical encoders of the DC servo motors are fed back into the 
servo drive amplifier and into the dSPACE. The readings from the link's strain gauge sensors are fed into strain gauge amplifiers and the dSPACE. Readings from the force sensor are fed into the force sensor kit amplifier, then into dSPACE. The controller was designed in MATLAB Simulink and runs together with the dSPACE software on the PC. The dSPACE 1103 controller board was used which has analog to digital channels (ADC) and digital to analog channels (DAC) to support device driving and communication. The schematic for this experimental setup is as shown in Figure 2.

When the force sensor is just touching the stand without exerting any force, this state is referred to as the restrained state. At the beginning of every experiment, the force sensor is at the restrained state. The stand had a height of $1015 \mathrm{~mm}$, and the height was determined so that the stand and the sensor would just make contact when Link 2 was horizontal. When joint 3 is rotated, link 2 rotates and the force sensor at its tip presses against the stand. The vertical downward direction in Figure 2 is defined as the z-axis positive, the horizontal left direction is defined as x-axis positive, and the inward depth direction is defined as $y$-axis positive, and the clockwise rotation motion of the link pushes the tip in the positive direction of the $\mathrm{z}$-axis is called normal rotation. The counterclockwise rotation that would pull back the tip is called reverse rotation. Our focus is to analyze the contact forces generated along the $\mathrm{x}, \mathrm{y}$, and $\mathrm{z}$-axis directions. By inputting the voltage according to the target or setpoint at the start of the experiment, only Joint 3 is rotated normally. Joint 2 has a holding brake function that tries to maintain a stationary state when a constant voltage is commanded.

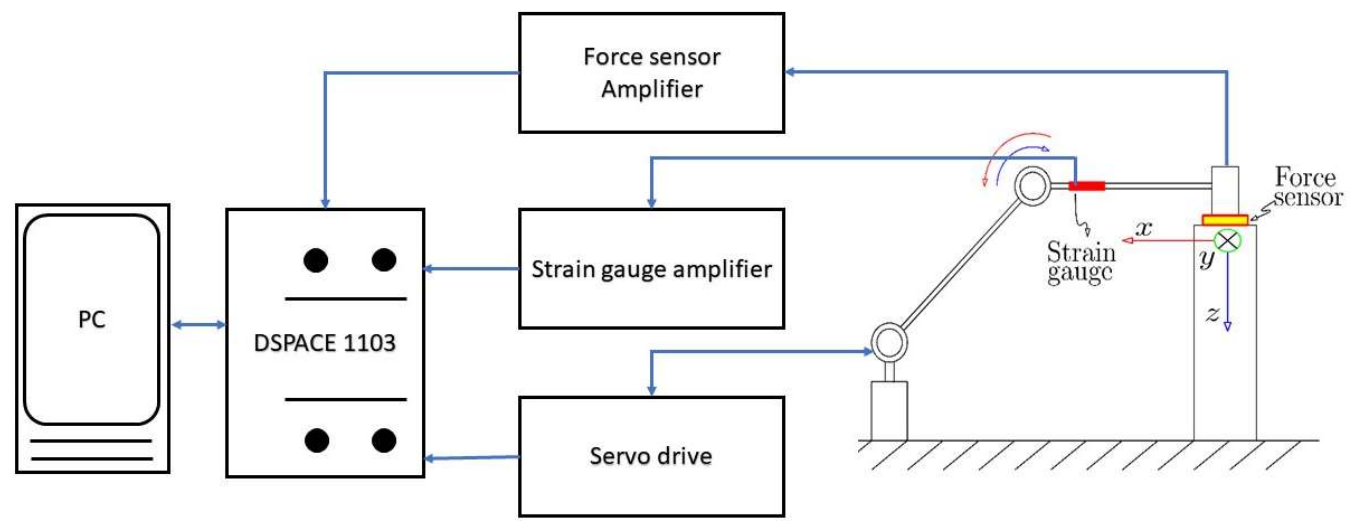

Figure 2. Schematic for the experiment setup

\section{Relational expression formulation and validation}

This section describes how the relational expression relating the root link strains and joint angles to the contact tip forces was empirically formulated. Later, these relations were experimentally validated using a uniformly increasing target force and a stepwise increasing target force.

\subsection{Derivation of contact force conversion formula}

At the beginning of the experiment, joint 3 is rotated in the normal direction by $3^{\circ}$. From these measurements, the relational expression between the angle of joint 3 and the contact force was derived. Figure 3 shows the changes in the contact forces in the $x, y$, and $z$ axes and link strains. The strain readings for both links were found to be off the zero or neutral point by some values, hence, processing was performed to restore the start position of the strain readings to the origin. From the strain readings, the strains for link 1 and link 2 were proportionally going in the negative direction with respect to time for the entire duration of the experiment. This was because Hooke's law holds in the range of the proportional limit for both aluminum and stainless steel. Both data oscillate near 5(sec) and 15(sec), this was attributed to the fact that the tip of the Joint 3 experienced slip phenomenon. The relations obtained here are shown in Table 2. $\varepsilon_{1}$ and $\varepsilon_{2}$ are the amount of strain at the root of link 1 and link2, respectively. $\theta_{2}[\mathrm{deg}]$ is the joint angle of joint 3 . 


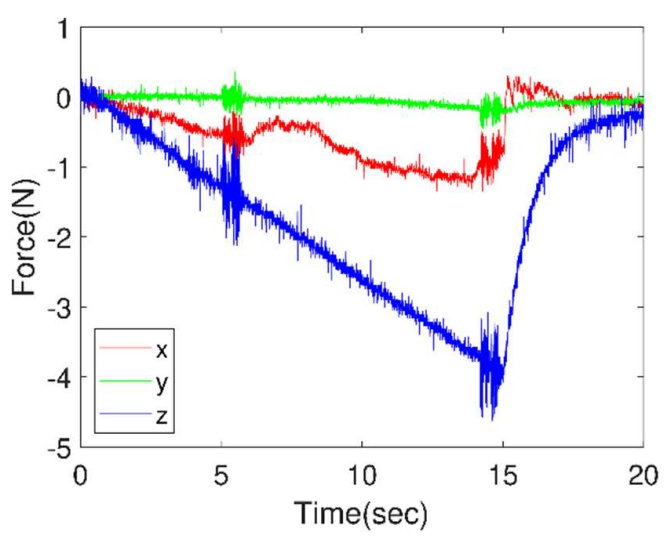

(a)

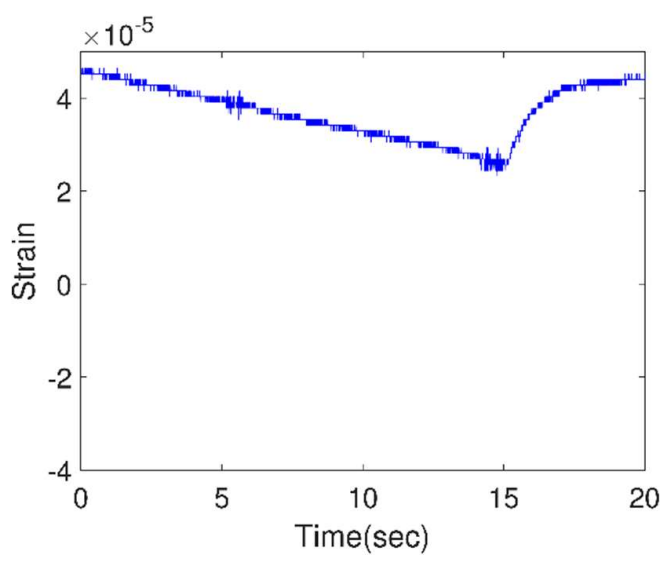

(b)

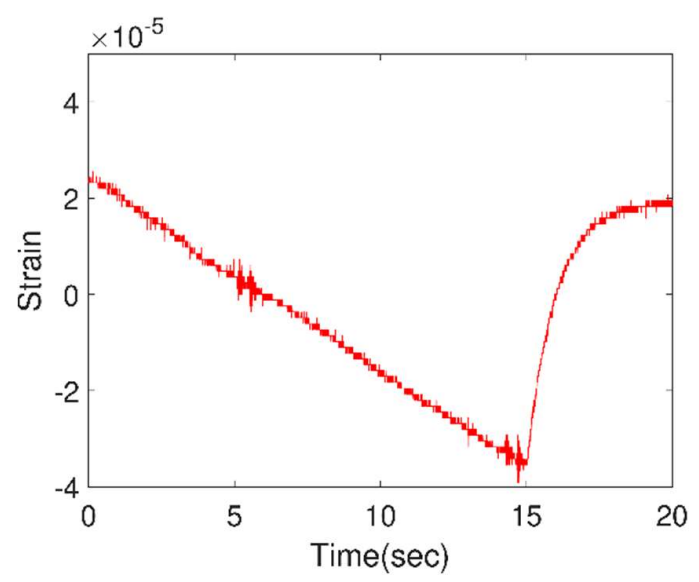

(c)

Figure 3. Force and strain (a) output tip force; (b) Strain, Link 1; (c) Strain, Link 2;

Table 2. Relational expression

\begin{tabular}{cr}
\hline parameter & relation \\
\hline$\varepsilon_{1}$ & $0.020 \times F$ \\
$\varepsilon_{2}$ & $0.061 \times F$ \\
$\theta_{2}$ & $0.700 \times F$ \\
\hline
\end{tabular}

\subsection{Verification of the relational expression}

\subsubsection{Continuous incremental force tracking}

Figure 4 shows a comparison of the strain amounts of Link 1 and Link 2 against force obtained using the relational expression derived from the contact force in the z-axis direction. From the results, since the respective strain amounts and the data for the contact force in the $\mathrm{z}$-axis direction agree, a proportional relationship was assumed. Therefore, for performing contact force control, the strain of Link 2, which has a larger output and more resolution than link 1 , and is closer to Joint 3 , which is the moving actuator, was used. In Figure 4, the contact force graphs were smoothed using a 50th-order median filter for improved visualization and comparison of the overall mean trend. 


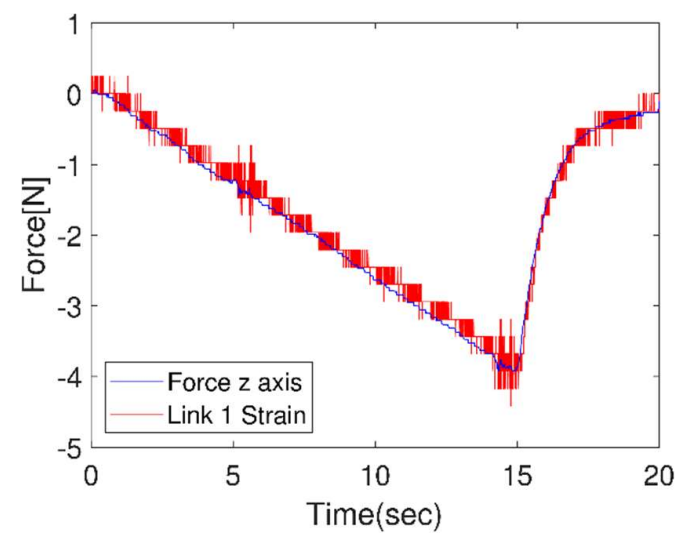

(a)

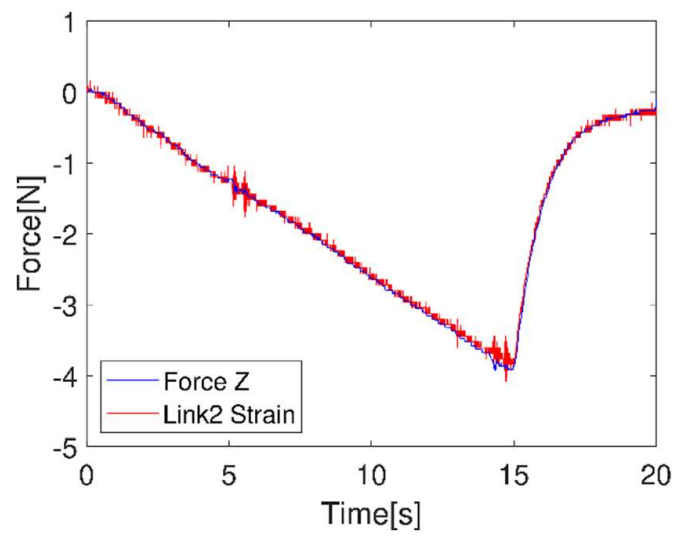

(b)

Figure 4. Force and strain (a) link 2; (b) Link 2.

\subsubsection{Incremental force tracking}

To investigate the validity of the angle/force relational expression presented in table 1, the angle of joint 3 was measured as the target tip force was varied. In this experiment, the target tip force was varied by $-1 \mathrm{~N}$ from the restrained state until a final value of $-3 \mathrm{~N}$. The actual tip force results are shown in Figure 5a where force is seen to increment in steps of $-1 \mathrm{~N}$. Also, the tip contact force in the x-axis direction was observed. Figure $5 b$ shows how the angle at joint 3 behaved. When the force was constant, the angle was constant too. The angle tracked the increments of the tip contact force. Figures $5 \mathrm{c}$ and $5 \mathrm{~d}$ show the relation of the link strain with the actual tip contact force for link 1 and link 2, respectively. Again, a 50th-order median filter was employed to smoothen the data for the z-axis tip contact force for better visualization of the median trend, to enable comparison with the link strains for the whole range of experiment. Even though the resolution of the link 1 strain is lower in Figure 5c, well tracking is observed. Tracking was observed also for link 2 strain data in Figure $5 \mathrm{~d}$. at $-3 \mathrm{~N}$ there was an error between the strain and the force value which we deemed acceptable. Since both strain and force data are compared to validate each other, and none of the two are calibrated, subsequently in our experiments, both data shall be presented.

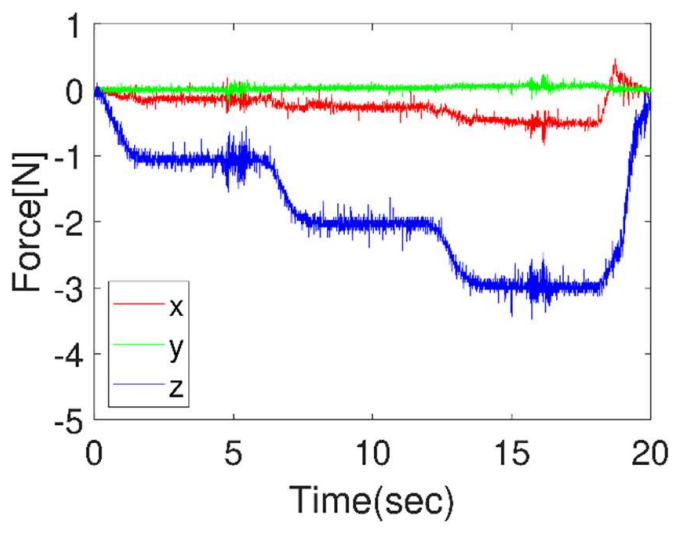

(a)

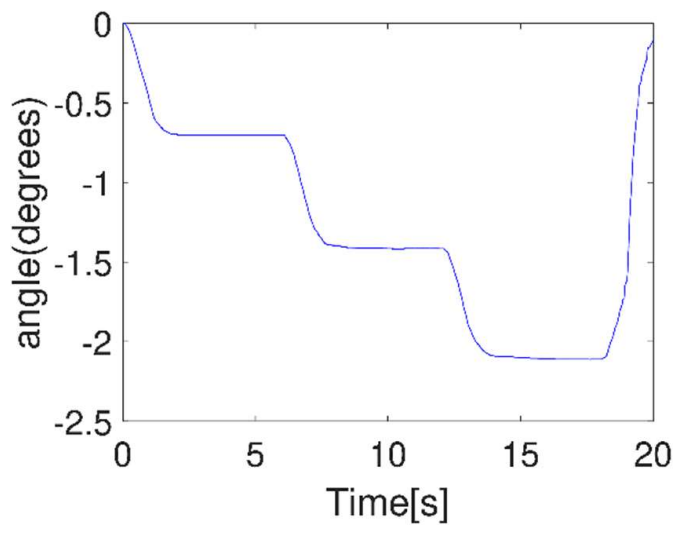

(b) 


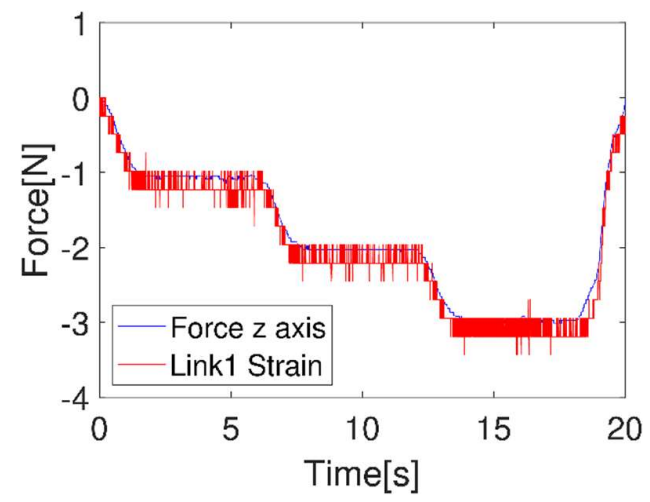

(c)

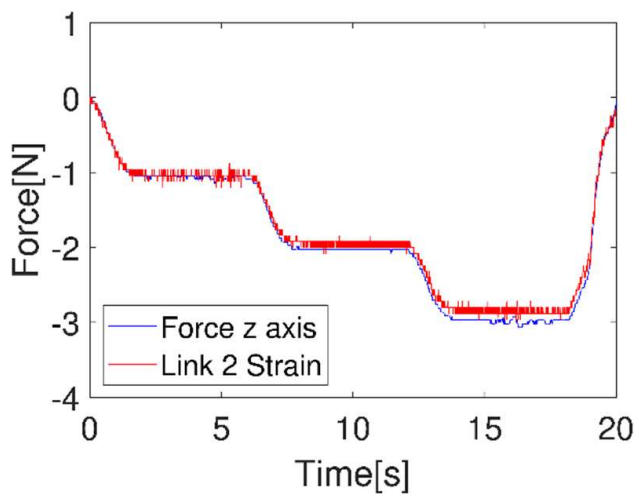

(d)

Figure 5. Force and strain tracking angle (a) Actual tip contact force; (b) commanded joint angles; (c) Link 1 strain versus median contact tip force; (d) Link 2 strain versus median contact tip force

\subsection{Force controller development}

\subsubsection{Strain feedback scheme}

Figure $\underline{6}$ shows a block diagram of the proposed contact force control system based on the link strain feedback scheme. First, the target contact force $F_{d}$ was converted to the target strain amount $\varepsilon_{d}$ using the relation expression from Table 1. Again, the relational expression was used to convert the strain amount to angle. Next, transformation is performed on the angles using the relational expression to generate the motor input voltage $\mathrm{V}$ for Joint 3 . Finally, the root strain of link 2 is fed back in a subtractive sense to track the target strain that was generated from the target contact force. This way, it was possible to track the target contact force by using a feedback scheme based on the link strain.

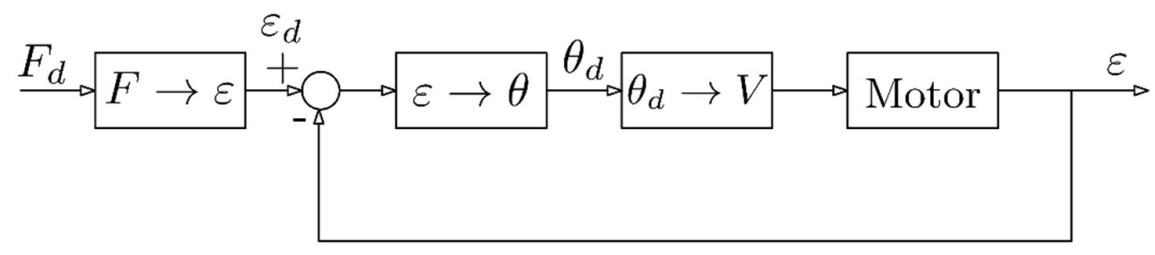

Figure 6. Strain feedback scheme

\subsubsection{Force feedback scheme}

Figure 7 shows a block diagram of the contact force feedback scheme. The actual z-axis direction contact force gathered by the force sensor is fed back and compared with the target contact force in a subtractive sense. The force difference is then converted to strain using the relation expression, the strain is converted to angle, the angle is converted to a voltage which drives the motor of joint 3. It is known that the force feedback method is the same as high gain destabilization [16], thus, the proportional gain that determines the angular velocity was set at 0.025 .

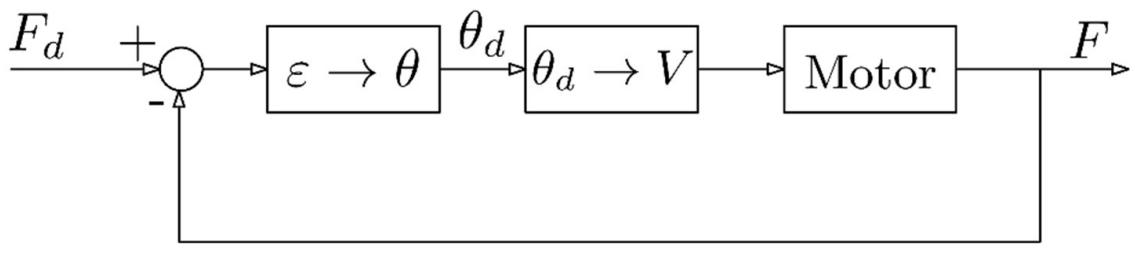

Figure 7. Force feedback scheme 


\section{Results and discussion}

Strain and force feedback control schemes were implemented experimentally, where the forces setpoints were $-1 \mathrm{~N},-2 \mathrm{~N}$, and $-3 \mathrm{~N}$, and the results shown in Figures 8, 9, and 10, respectively. For all the cases here, the gain for gravity compensation was 3 . Voltage is fed into the motor 1 second after the start of the experiment and a reverse rotation operation is performed at 30 seconds. The experiment is stopped at 35 seconds.

\subsection{Strain feedback control}

Throughout the contact force control results, the contact force in the z-axis direction follows the target value as it rises and settles at around 5 seconds for the $-1 \mathrm{~N}$ target force. Sudden oscillations of force values were observed for all the data for Figures 8, 9, and 10. This was attributed to the slip phenomenon. For instance, for the $-1 \mathrm{~N}$ case (Figure 8a), the slip was observed at the beginning and around 15- and 30-seconds instances, for all the three axes. The explanation for the slip is as follows; At first, when the tip is just contacting the stand, the tip is vertically positioned, and no force is pushing it in any direction. At this juncture the link 2 is horizontal. When the motor rotates, and the link tip contacts the stand, and the force progresses from zero upwards. Link 2 starts to elastically bend downwards and pulls the link's tip in both the positive x-axis direction and positive z-axis direction. At the initial contact when the downward force is applied, static friction constant prevails, at slip duration, the kinetic friction coefficient prevails, which is lower than the static friction coefficient. But the z-axis force is dominant and pushes the tip vertically downward, restoring the static coefficient dominance. This push and pull act results in oscillations, which are recorded by the strain sensor and fed back to the comparator. Each slip duration was about 2 seconds.

Figure $8 \mathrm{~b}$ shows the median filtered data of Figure $8 \mathrm{a}$ to enable clear observation of the trend and amplitude of the actual tip force. Figure $8 \mathrm{~b}$ shows the comparison of link 1 strain data with the median output force for the z-axis direction. The link 1 strain tracked the force data for all the experiment duration (Figure 1c) however the strain resolution was smaller than for link 2. Figure $8 \mathrm{~d}$ shows the relation of link 2 strain with the actual tip median force where slip was seen on link 2 strain data.

A similar set of events and trends that were observed in Figure 8 for the $-1 \mathrm{~N}$ case are also observed for the $-2 \mathrm{~N}$ case (Figure 9) and $-3 \mathrm{~N}$ case (Figure 10). The strain feedback enabled the actual z-axis force output to reach the $-2 \mathrm{~N}$ (Figure 9a) and $-3 \mathrm{~N}$ (Figure 9b) target values. The slip phenomenon was observed. A larger value of $\mathrm{x}$-axis force was observed, where a value of $-1 \mathrm{~N}$ was observed for $-3 \mathrm{~N}$ case as seen in Figure 10a. Figure $9 \mathrm{~b}$ and 10b give the median values for Figures 9a and 10a, respectively. For $-2 \mathrm{~N}$ target force, settling happens at about $7 \mathrm{sec}$. For $-3 \mathrm{~N}$ target force, settling to the final value is observed at about 8 seconds.

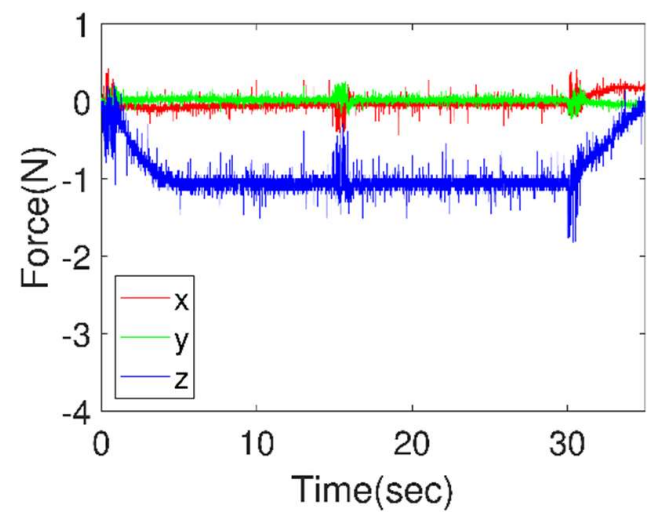

(a)

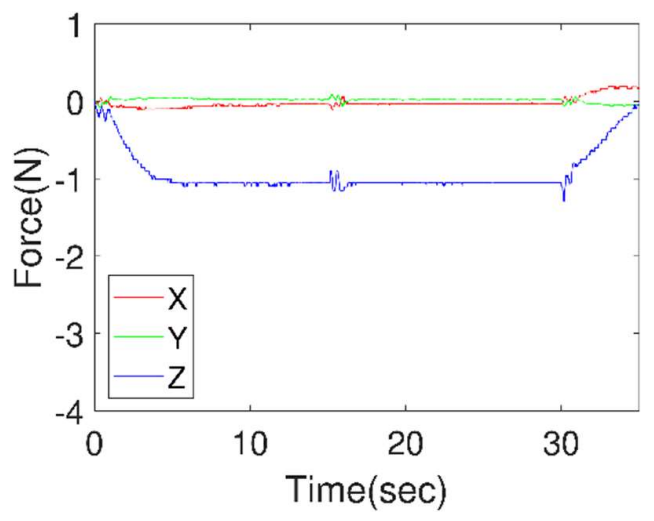

(b) 


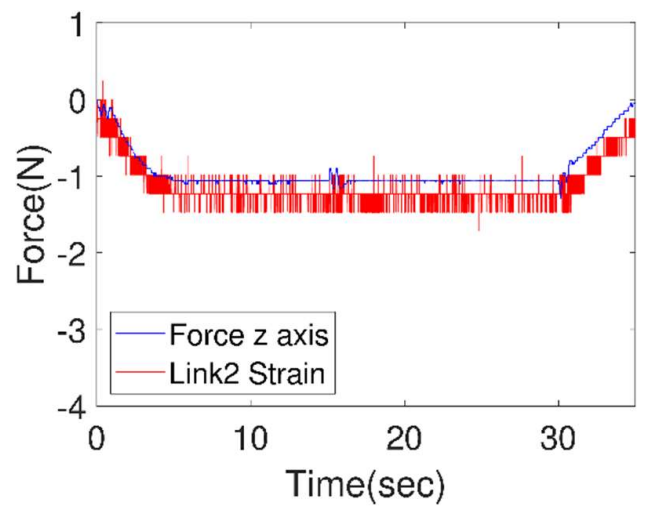

(c)

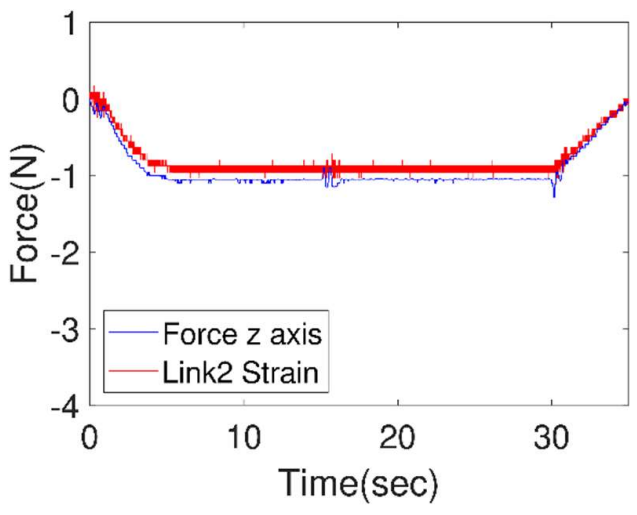

(d)

Figure 8. Strain feedback method, desired force = -1N; (a) Actual tip contact force; (b) median filtered data for (a); (c) Link 1 strain versus median contact tip force; (d) Link 2 strain versus median contact tip force

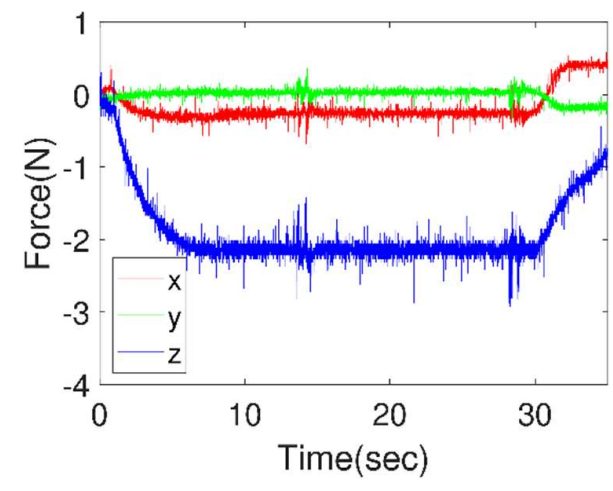

(a)

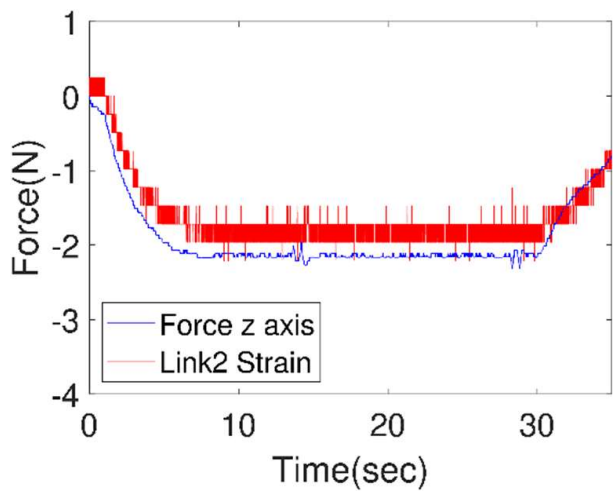

(c)

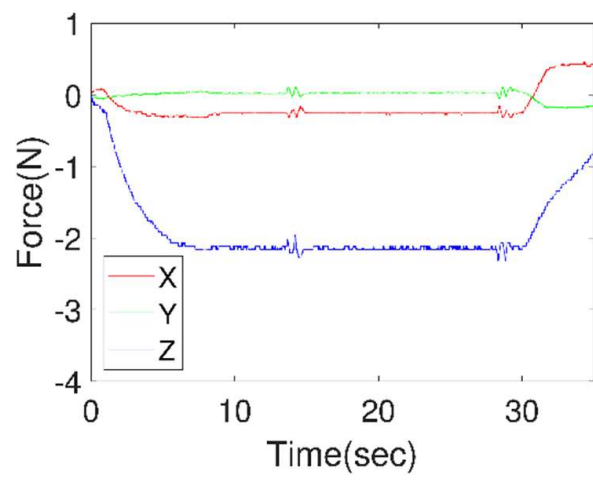

(b)

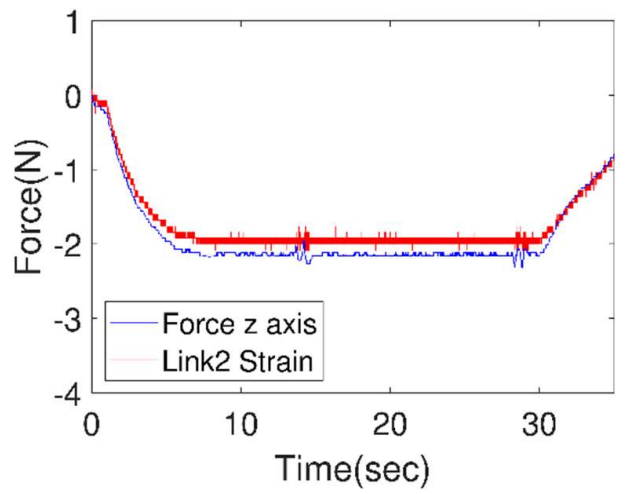

(d)

Figure 9. Strain feedback method, desired force = -2N; (a) Actual tip contact force; (b) median filtered data for (a); (c) Link 1 strain versus median contact tip force; (d) Link 2 strain versus median contact tip force 


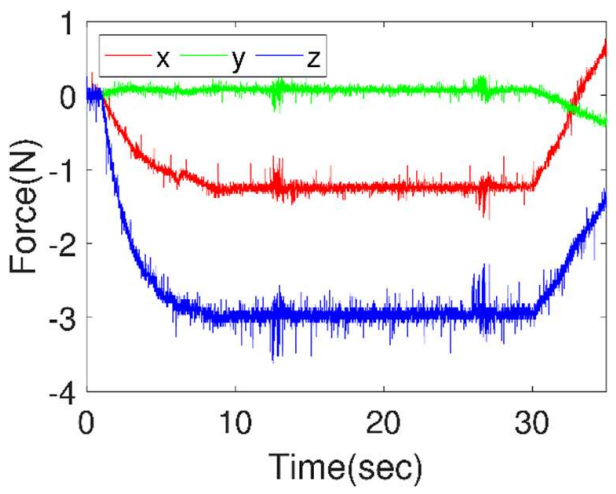

(a)

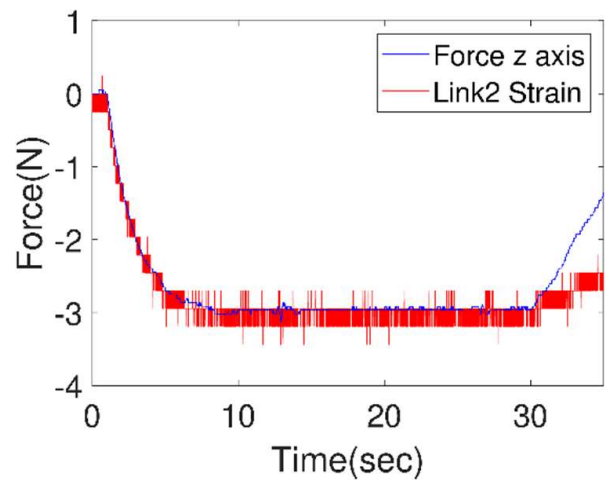

(c)

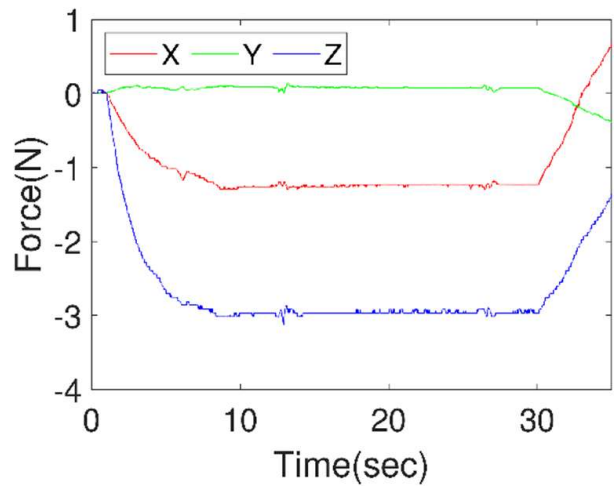

(b)

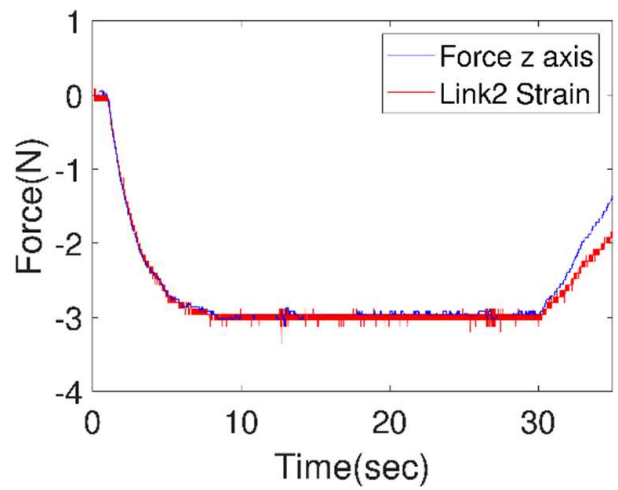

(d)

Figure 10. Strain feedback method, desired force = $-3 \mathrm{~N}$; (a) Actual tip contact force; (b) median filtered data for (a); (c) Link 1 strain versus median contact tip force; (d) Link 2 strain versus median contact tip force

\subsection{Force feedback control}

To investigate the effectiveness of the force feedback scheme, the output tip force was measured and compared with strain at link 1 and link 2 . The results for the force feedback method are shown in Figures $\underline{11} \underline{12}$, and $\underline{13}$. The actual contact force z-axis direction follows the target force value of -1N (Figure 11a), -2N (Figure 12a), and -3N (Figure 13a). The median data for these actual contact forces for the $x, y$, and $z$ axes are shown in Figure 11b, 12b, and 13b. These median figures do not show the high-frequency data but it enables one to see the overall trend. These median data were used for comparison to the root link strain data for link1 (Figure 11c, 12c, and 13c) and link 2 (Figure 11d, $12 \mathrm{~d}$, and 13d). For all these strain results, the median output force data well tracked the strain data meaning the relation expression worked well within the controller. The slip phenomenon was observed where the controller maintained on the commanded track well after the slip event passed. Since the controller kept track of the setpoint after the slip event, then this force controller is a viable choice. 


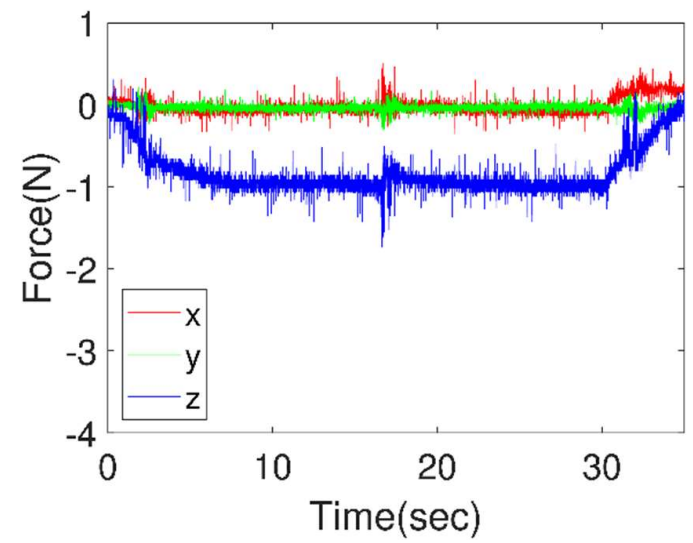

(a)

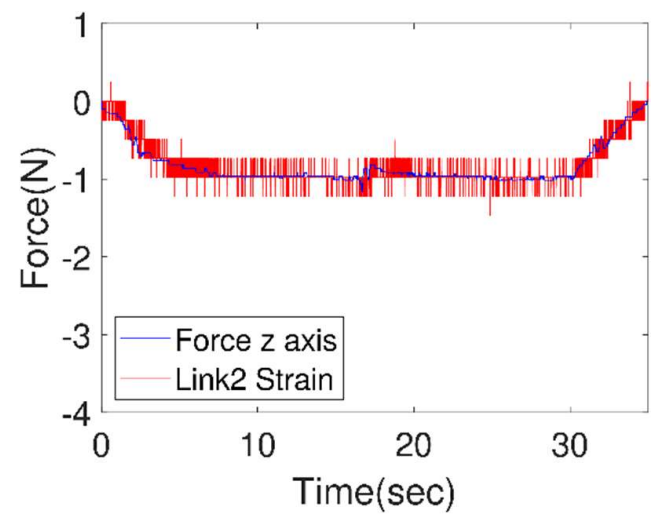

(c)

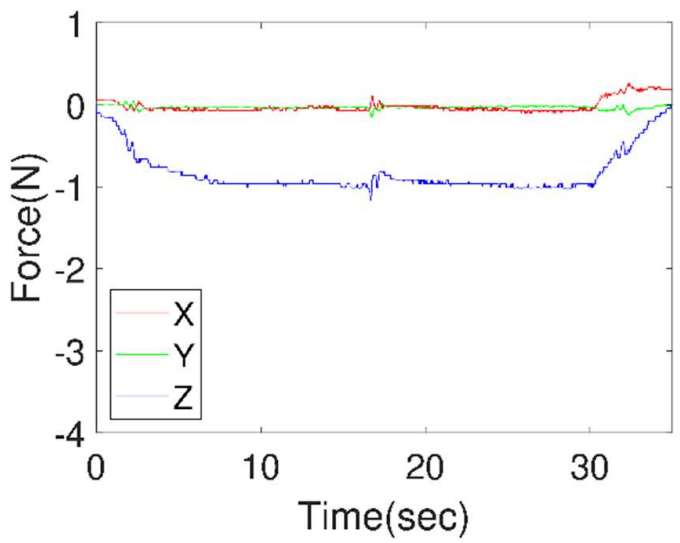

(b)

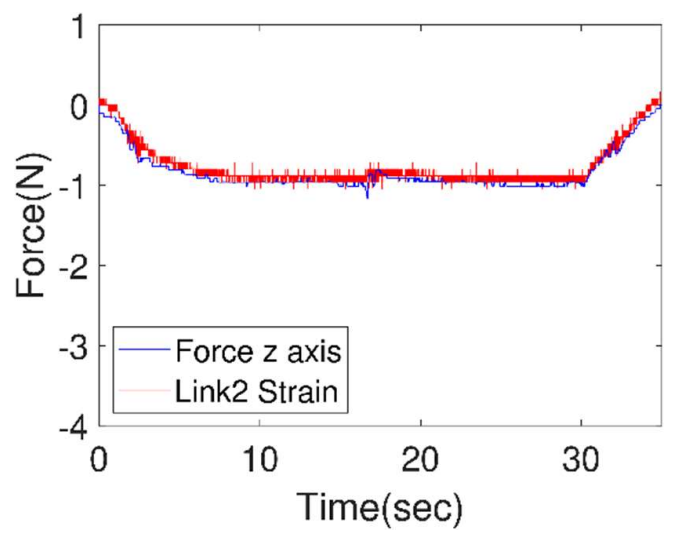

(d)

Figure 11. Force feedback method, desired force = -1N; (a) Actual tip contact force; (b) median filtered data for (a); (c) Link 1 strain versus median contact tip force; (d) Link 2 strain versus median contact tip force

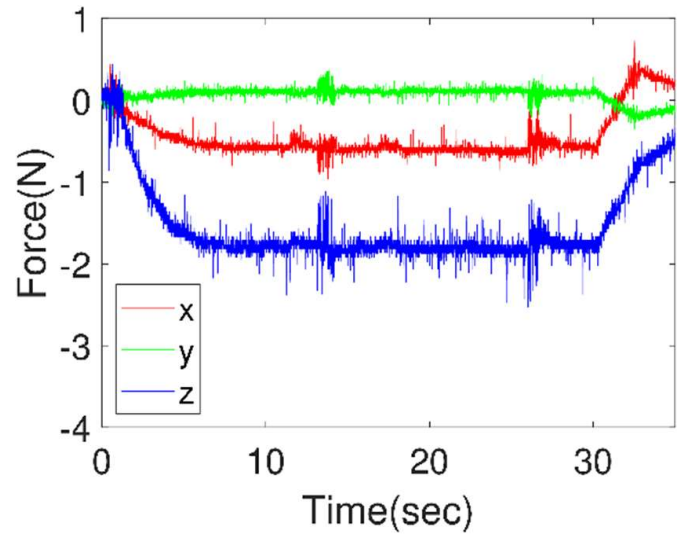

(a)

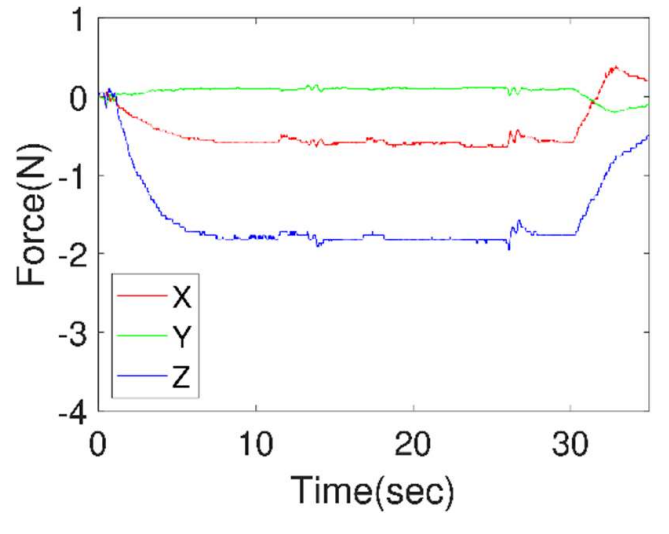

(b) 


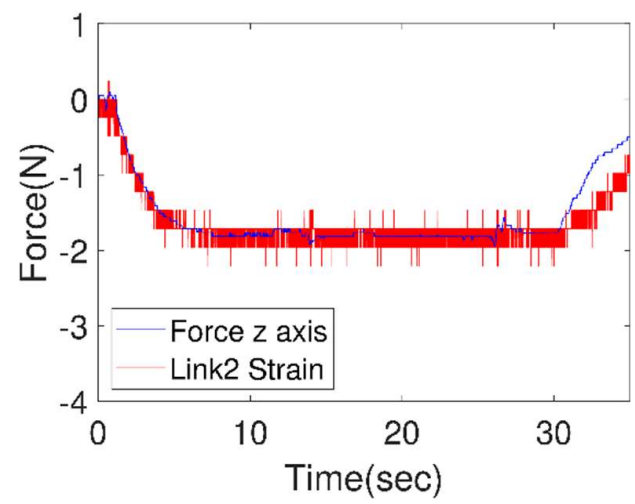

(c)

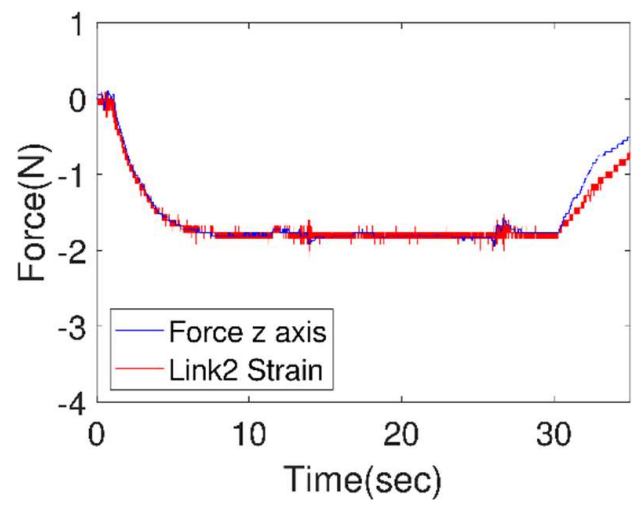

(d)

Figure 12. Force feedback method, desired force =-2N; (a) Actual tip contact force; (b) median filtered data for (a); (c) Link 1 strain versus median contact tip force; (d) Link 2 strain versus median contact tip force

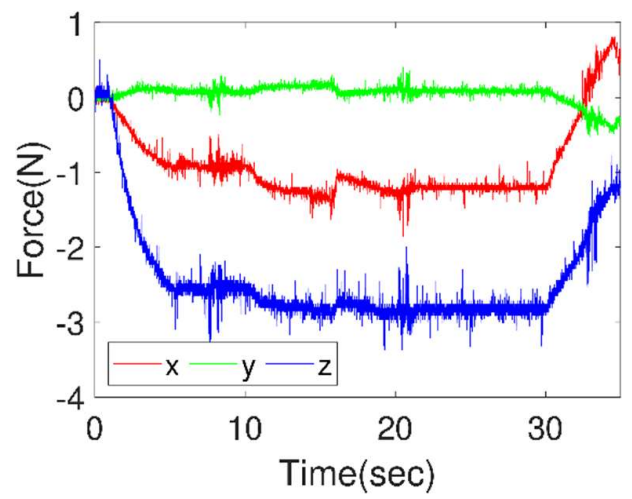

(a)

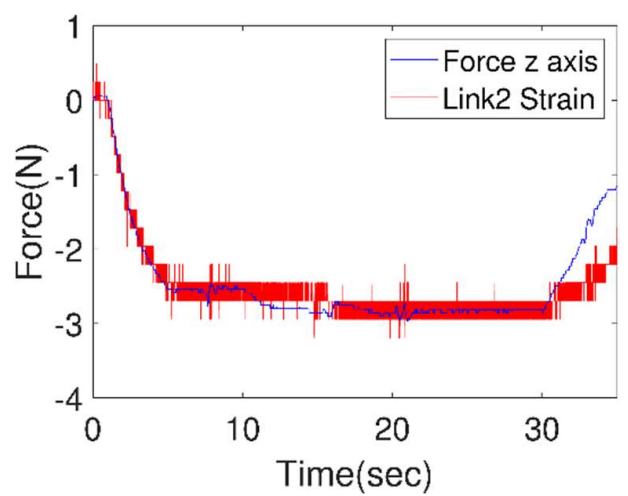

(c)

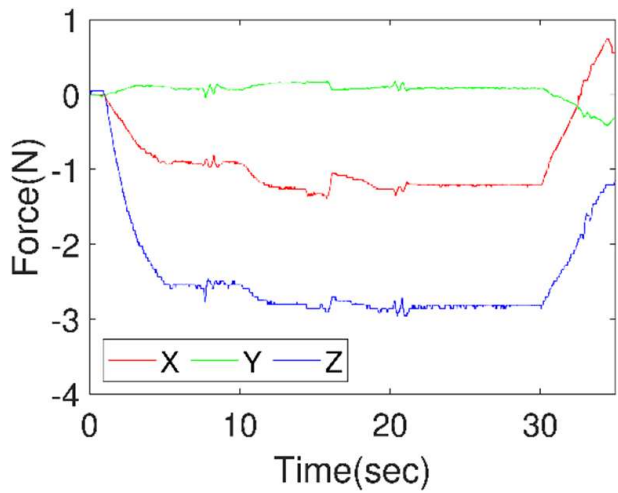

(b)

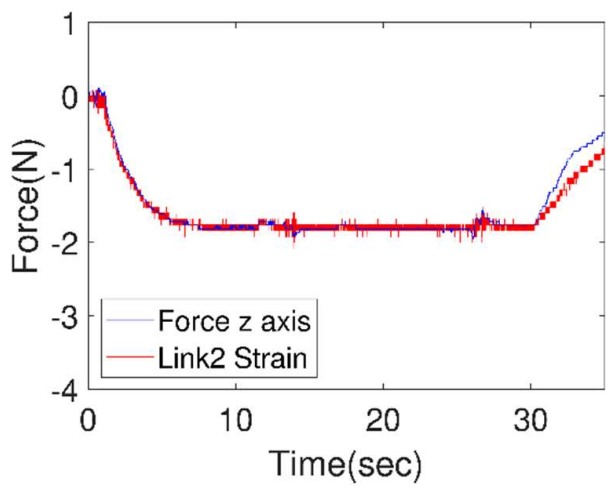

(d)

Figure 13. Force feedback method, desired force = -3N; (a) Actual tip contact force; (b) median filtered data for (a); (c) Link 1 strain versus median contact tip force; (d) Link 2 strain versus median contact tip force

\subsection{Comparison of performance of strain and force feedback control schemes}

Figure 14 shows a comparison of the median filtered performance for the force outputs of the two feedback methods where they both attained the target mean positions of $-1 \mathrm{~N},-2 \mathrm{~N},-3 \mathrm{~N}$. The slip events can be observed in all the data and they occurred at different times, where the slip at the tip was transmitted to the root of link 2 . Since slip is a function of the dynamically changing contact surfaces and the force vector, elimination of the slip artifact was not the objective of this controller. Better yet, both the control schemes enabled quick recovery to track the setpoints after the slip event 
passed. Therefore, the performance of both these controllers was satisfactory since they well tracked the target force. The comparison of these two controller schemes is of importance since the strain gauges and the force sensors are not factory calibrated for this flexible manipulator.

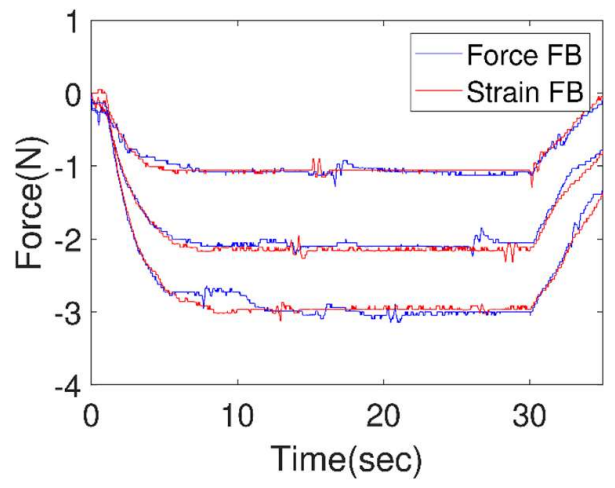

\section{Conclusion}

Figure 14. Comparison of strain and force feedback schemes

Force control of a 2-link spatial flexible manipulator using a force feedback scheme was experimentally demonstrated and validated using a strain feedback scheme. The link's root strain, the joint angles, and the tip contact forces were experimentally obtained and the relational expression for these three quantities was obtained empirically. Both controllers tracked the target force values of $1 \mathrm{~N},-2 \mathrm{~N}$, and $-3 \mathrm{~N}$. The slip phenomenon was observed where the controllers continued to track even after the slip events ceased. Simple force control strategies are a promising approach to enable the implementation of low weight flexible manipulators to function as industrial robots.

Author Contributions: conceptualization, MS and IS; methodology, DM and WN; software, KM; validation, MS, JKM, and TM; project administration, MS, and KM.

Acknowledgments: This work is partially supported by Grants-in-aid for Promotion of Regional Industry-University-Government Collaboration from Cabinet Office, Japan.

Conflicts of Interest: The authors declare no conflict of interest.

\section{References}

[1] W. Njeri and M. Sasaki, “Two degree-of-freedom vibration control of a 3D, 2 link flexible manipulator," 2018.

[2] T. H. Lee, S. S. Ge, and Z. P. Wang, "Adaptive robust controller design for multi-link flexible robots," Mechatronics, vol. 11, no. 8, pp. 951-967, 2001, doi: 10.1016/S0957-4158(00)00062-3.

[3] S. S. Ge, T. H. Lee, and G. Zhu, "Energy-based robust controller design for multi-link flexible robots," Mechatronics, vol. 6, no. 7, pp. 779-798, 1996.

[4] Z.-H. Luo, "Direct strain feedback control of flexible robot arms: new theoretical and experimental results," IEEE Trans. Automat. Contr., vol. 38, no. 11, pp. 1610-1622, 1993.

[5] Z. H. Luo, F. Matsuno, and Y. Sakawa, "Experimental study on feedback control of coupled bending and torsional vibrations of flexible beams," in Control of Distributed Parameter Systems 1989, Elsevier, 1990, pp. 327-332.

[6] H. G. LEE, F. MIYAZAKI, and S. ARIMOTO, "PDS feedback control for flexible multi-link manipulators," Trans. Soc. Instrum. Control Eng., vol. 25, no. 6, pp. 675-681, 1989.

[7] F. Matsuno and K. Yamamoto, "Dynamic hybrid position/force control of a two degree-of-freedom flexible manipulator," J. Robot. Syst., vol. 11, no. 5, pp. 355-366, 1994.

[8] L. Liu and K. Yuan, "Force control of a constrained one-link flexible arm: A distributed-parameter modeling approach," J. Chinese Inst. Eng., vol. 26, no. 4, pp. 443-454, 2003. 
[9] H. KOJIMA and T. KAWANABE, "Position and Force Control of a Flexible Robot Arm with PIS Control," J. Robot. Soc. Japan, vol. 10, no. 3, pp. 353-360, 1992.

[10] T. Endo, M. Sasaki, F. Matsuno, and Y. Jia, "Contact-Force Control of a Flexible Timoshenko Arm in Rigid/Soft Environment," IEEE Trans. Automat. Contr., vol. 62, no. 5, pp. 2546-2553, 2017, doi: 10.1109/TAC.2016.2599434.

[11] T. Endo, F. Matsuno, and H. Kawasaki, "Force control and exponential stabilisation of one-link flexible arm," Int. J. Control, vol. 87, no. 9, pp. 1794-1807, 2014.

[12] T. Endo, M. Sasaki, and F. Matsuno, "Contact-force control of a flexible Timoshenko arm," IEEE Trans. Automat. Contr., vol. 62, no. 2, pp. 1004-1009, 2016.

[13] W. Njeri, M. Sasaki, and K. Matsushita, "Gain tuning for high-speed vibration control of a multilink flexible manipulator using artificial neural network," J. Vib. Acoust., vol. 141, no. 4, 2019.

[14] W. Njeri, H. Ngetha, M. Sasaki, N. Honda, and K. Matsushita, "Gain tuning using neural network for contact force control of flexible arm," 2020.

[15] G. Ferretti, G. Magnani, and P. Rocco, "On the stability of integral force control in case of contact with stiff surfaces," 1995.

[16] C. An and J. Hollerbach, "Kinematic stability issues in force control of manipulators," in Proceedings. 1987 IEEE International Conference on Robotics and Automation, 1987, vol. 4, pp. 897-903. 